\title{
Stability of word order: Even simple questions need careful answers
}

\author{
MARK DONOHUE
}

\section{Introduction}

Dunn et al. (2011) attempt an exciting piece of research, investigating which word-order parameters are co-dependent or independent, and which are stable or not. Claims about "universality" have to be taken with several grains of salt, since they investigate only four families, compared to the dozens of families that have been part of samples for the last several decades. More significantly, however, is the implicit claim that evolutionary models are appropriate for modelling the kinds of change (or stability) that they investigate. Since the focus of their article is on the family-internal patterns of conservatism or innovation in word order, I shall focus on an important aspect of their construction of language family relationships that appears to be at odds with the kinds of evolutionary models that they employ, and so which, failing to take into account contact (e.g., Thomason \& Kaufman 1988 and many more), reveals the study to suffer from a failure to consider critical data and models that are in fact more explanatory than their own.

\section{False family trees}

\subsection{True and false}

Citing a need for "quantitative phylogenies" (essentially, branches with quantified distances rather than simply topological relationships) in their construction of family relationships, Dunn et al. take the unusual step in employing trees constructed by reference not to normal historical linguistic principles, but by reference to lexical cognacies. It is well known that lexical items are the element of language most subject to borrowing, constrained by social distance (see, among many others, Cavalli-Sforza \& Wang 1986, Donohue, Denham \& Oppenheimer forthcoming, Goebl 1984, Hess 1979, Kessler 2001, Séguy 1971). Indeed, within the families that Dunn et al. discuss we have documented 
cases of up to $48 \%$ of the basic vocabulary of a language being borrowed from another family (e.g., Cham; Grant 2005), levels far in excess of levels that have been cited as the upper limits of reliability for the method that Gray uses (Gray et al. 2009 cite a high figure of $\sim 20 \%$ ), and frequently (at least in the case of the Austronesian data, with which I am most familiar) undetected as loans. As a result, the trees they measure model contact at least as much as they model historical descent, and so calibrating an evolutionary model against these yardsticks models susceptibility to (unusual?) social processes, rather than modelling phylogenetic stability. This is clearly not an appropriate metric against which to test the phylogenetic stability of typological features.

Some of the most gratuitous failings of the topography of Dunn et al.'s family trees, examining just Indo-European and Austronesian in detail, are discussed below. While I am aware that the trees presented in Dunn et al. are only some of the possible trees generated by their methodology, if they are representative of the trees generated (and if not, why publish them?) then they must be subject to critical examination.

\subsection{Indo-European}

In contrast to the usual depiction of Indo-European as involving a "rake" at the highest levels (though see, e.g., Ringe et al. 2002, Nakhleh et al. 2005, amongst others), the tree in Dunn et al. shows numerous low-level groups that are not widely established using the comparative method. We see Germanic and Romance subgrouped together, as well as Greek and Albanian, and Celtic together with Balto-Slavic and Indo-Iranian. Ancient languages are treated as "equivalent" to modern languages for subgrouping purposes, leaving us with the result that Ancient Greek is a sister to Modern Greek, not a precursor, and Modern English is no closer topologically to Old English than Old English is to (modern) German. If the structure, relative distance, and overall topology of the tree in the left of Figure 1 helps Dunn et al. to evaluate the relative stability of the two word order features they examined, then we can have little faith in the method. Figure 1 shows the overall topography of their Indo-European tree. It rather felicitously replicates the existence of known first-order subgroups of Indo-European, but fails to reproduce them as first-order subgroups, or as subgroups with widespread acceptance. Given the heavily embedded structure of Dunn et al.'s tree, compared to the widely accepted "rake", it is hard to see how any results arising from the hierarchical tree can have any meaning to the accepted tree. For instance, given that Dunn et al. examine word order change and find SOV orders to be present at the top of their tree (Hittite, Tocharian, Armenian) and at the bottom (Indo-Iranian), they have a claim for long-term stability through many intervening nodes from the proto-language to the modern Indic languages. Examining the occurrence of SOV order on a more con- 

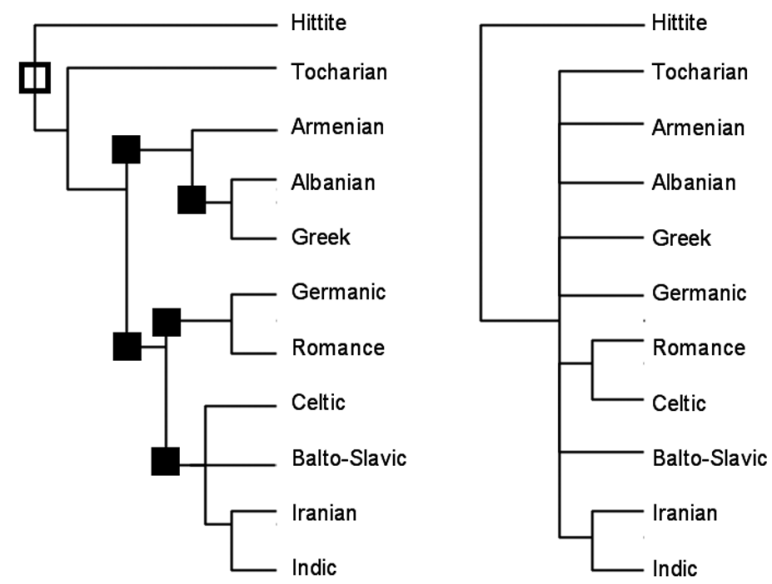

Figure 1. Dunn et al's subgrouping of Indo-European compared to a conventional tree (divisions that are more or less accepted are shown with an open square; divisions that are not widely accepted on the basis of comparative method proof are shown with a solid black square, e.g., the Albanian-Greek subgroup, or Germanic-Romance)

ventional tree (shown with boxes), however, we can see that no such claim is supported.

What causes this misrepresentation of the Indo-European tree? If the method examines lexical cognacy, and takes no account of sound change - thus emerging as a "mindless gimmick", following von der Gabelentz (1901: 168) - then it will create contact maps showing the networks of borrowing and retention (equally, given their methods, it will generate "uncontact" maps, indicating outliers which do not participate in a particular range of lexical sharing). Meillet (1908: 128-130) noted that many apparent cognates could be found in the vocabularies of the Germanic and Italo-Celtic groups but nowhere else in the Indo-European family (e.g., Latin piscis, Gothic fisk, Old Irish iasc 'fish'), but did not consider them reliable tests for historical relationships. By relying on trees reconstructed from this kind of unreliable data, Dunn et al. render their complicated assessments of stability invalid, since they do not measure stability against a path of inheritance. Since the Indo-European data they examine is considered in isolation from other families (true of all four of the families they examine), this means that they only be considering family-internal relationships; but with the wrong data, since they are not modelling the inherited aspects of the languages' history. 


\subsection{Austronesian}

The tree presented bears little relationship to commonly accepted views of Austronesian historical relations (as expressed in, for instance, Adelaar 2005a, b; Blust 1981, 2009; Lynch et al. 2002; Marck 2000; Pawley 1972; Ross 1988, 1995), and bears a strong resemblance to a geographical clustering of languages branching out from a centre of diversity in Taiwan (hence the somewhat successful replication of low-level subgroups within the family, which generally spread into geographically contiguous areas). The "spine" of the tree contains seventeen nodes between the start and the Polynesian languages at the geographic end; of these seventeen, four represent nodes that are accepted by Austronesian historical linguists (shown with unfilled squares: MalayoPolynesian, Eastern Malayo-Polynesian, Oceanic, and Central Pacific), and one other node represents a commonly accepted subgroup (Western Oceanic, containing the Schouten, North New Guinea, Papuan Tip, and Meso-Melanesian languages). The rest of the clusters are not phylogenetic, and represent in the main geographic clusterings. For instance, the Philippines languages and Bajo (from the Barito group) cluster together because of the presence of Bajo at the southern end of the Philippines; Irarutu, from the South Halmahera/West New Guinea group, clusters with Central Maluku because of its position on the west of New Guinea facing, and in contact with, languages of Central Maluku; the Admiralties group of Oceanic clusters with the Western Oceanic languages because it is surrounded by them and in contact with them, while the North-West Solomonic languages (part of Meso-Melanesian) are split from their northern sisters by Bougainville, a non-Austronesian stronghold in the centre of the Meso-Melanesian range; and Tongan and Samoan group together, and not with their close relatives, due to extensive contact (and conservatism) between and within both languages (Donohue et al. forthcoming).

While the accepted trees for Indo-European and Austronesian do not involve binary branches, which are apparently more easily amenable to statistical treatment of the type that Dunn et al. employ, it is nonetheless a mistake to consider binary branching an appropriate model of language diversification, and an algorithm that necessarily produces binary branching trees is simply not appropriate. Work on the early structure (or lack of it) for certain Indo-European groups reveals the perils involved in trusting to the categorial nature of linguistic subgroups (Garret 2006), and similar cautions should be applied to Austronesian (e.g., Blust 2009, Donohue \& Denham 2010) and Uto-Aztecan (see the debate on the appropriateness of a north-south division in the family in, for instance, Campbell 1997, Goddard 1999, and Mithun 1999) and Bantu (for which no experts agree on anything but a rake-like dispersal for most of the languages). If the methodology cannot easily accommodate itself to a probably more realistic model of language differentiation, then it is an inappropriate methodology. 

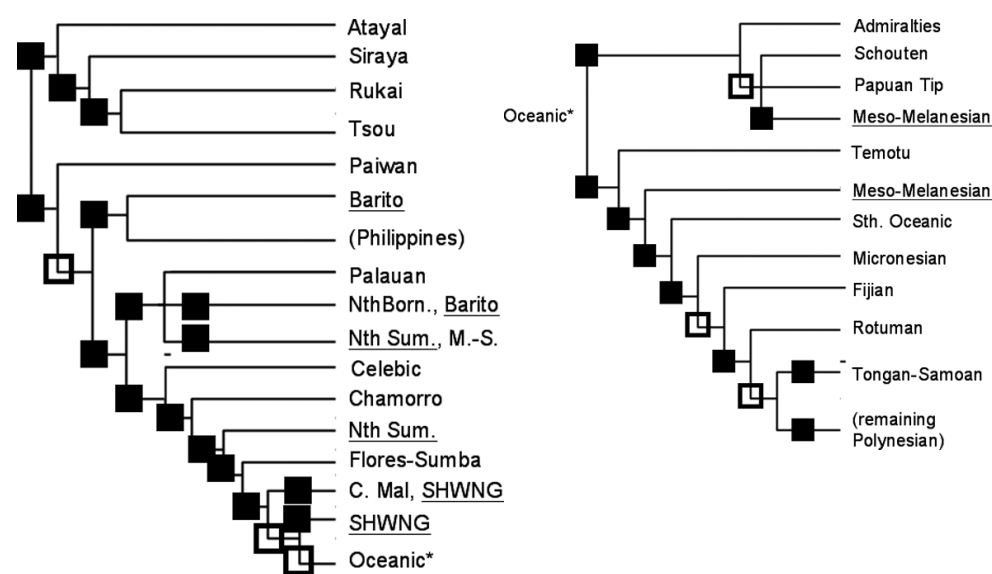

Figure 2. Dunn et al's topography of Austronesian language relationships (Nth Born. $=$ North Borneo, Nth Sum. $=$ North Sumatra/Barrier Islands, M. - S. = Malayo-Sumbawan, C. Mal = Central Maluku, SHWNG = South Halmahera/West New Guinea; the Oceanic node shown at the bottom of the figure on the left is continued on the right; underlined subgroup names represent subgroups split over more than one of Dunn et al.'s nodes)

\section{Inappropriate isolation}

In Dunn et al.'s study each family is modelled in isolation from its social contexts with other families. Consider: Indo-European has been in contact with at least 15 families for centuries: Afro-Asiatic, Austro-Asiatic, Basque, Burushaski, Dravidian, Eskimo-Aleut, Finno-Ugric, Kartvelian, Kusunda, Mongolic, Nakh-Daghestanian, North-West Caucasian, Tibeto-Burman, Tungusic, and Turkic. While Dunn et al. do consider four different families, they are four families that have not been in contact with each other until recent historical times, and so do not constitute a sample that allows us to investigate contact effects. Despite this Dunn et al. proceed using a model that is sensitive to contact effects, meaning that we have access to only a fraction of the relevant information. The preservation of SOV order among Indo-European languages in South Asia, combined with its almost total loss elsewhere, cannot be unrelated to contact with SOV-dominated Dravidian (and other) languages in that region. Similarly the presence of a small collection of SOV languages in the Austronesian family that are found on the coasts of eastern New Guinea cannot be reasonably discussed without reference to the non-Austronesian languages of New Guinea, among which SOV order is predominant (Foley 1986, Ross 2001, Donohue 2010). 
We have seen that the model of the family trees for Indo-European and Austronesian bears little resemblance to known (or even speculated) genealogical relations. Even more importantly, only one region in the Austronesian tree shows a concentration of fully-blown shift to OV (and other head-final) patterns, the part that represents the languages of south-east New Guinea. These languages have long been acknowledged to have undergone large levels of contact-induced change (Ross 1988, 2001, 2011; Lynch et al. 2002), probably the result of language shift on the part of the local populations. Why, then, do we believe that change in word order follows from PHYLOGENETIC principles, and a top-down modelling, as opposed to looking for family-external explanations? Given what is known about the ease of diffusion of linguistic traits (e.g., Thomason \& Kaufman 1988, Holman et al. no date), the failure to consider contact as a possible influence on morphosyntax (either by providing support for a conservative word order, or incentives to innovate) is remarkable.

To illustrate this point forcefully, consider the following maps (Dryer 2011). In Map 1 we note that all occurrences of SOV order in Austronesian languages are found in languages spoken on coasts along the eastern half of New Guinea. Map 2 shows that these languages are contiguous with the vast bulk of SOV languages found in New Guinea, not of the Austronesian family but nonetheless exerting a strong areal pressure. The Austronesian languages without verbfinal order are all found in regions where there is not a strong non-Austronesian presence to enforce an SOV word order, allowing the conservative verb-initial order, or a verb-medial order (itself a contact-induced change; Donohue 2005, 2007) that was inherited from Proto-Austronesian (and Proto-Oceanic). The in-

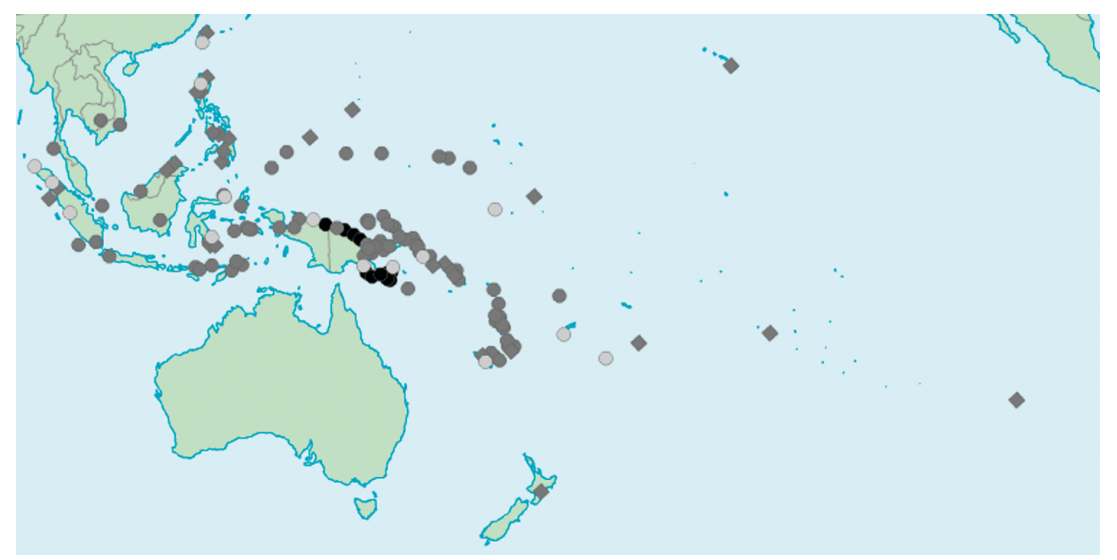

Map 1. Austronesian languages form WALS with SOV word order (black, on New Guinea) compared to SVO (grey circles) and V-initial (grey diamonds) 


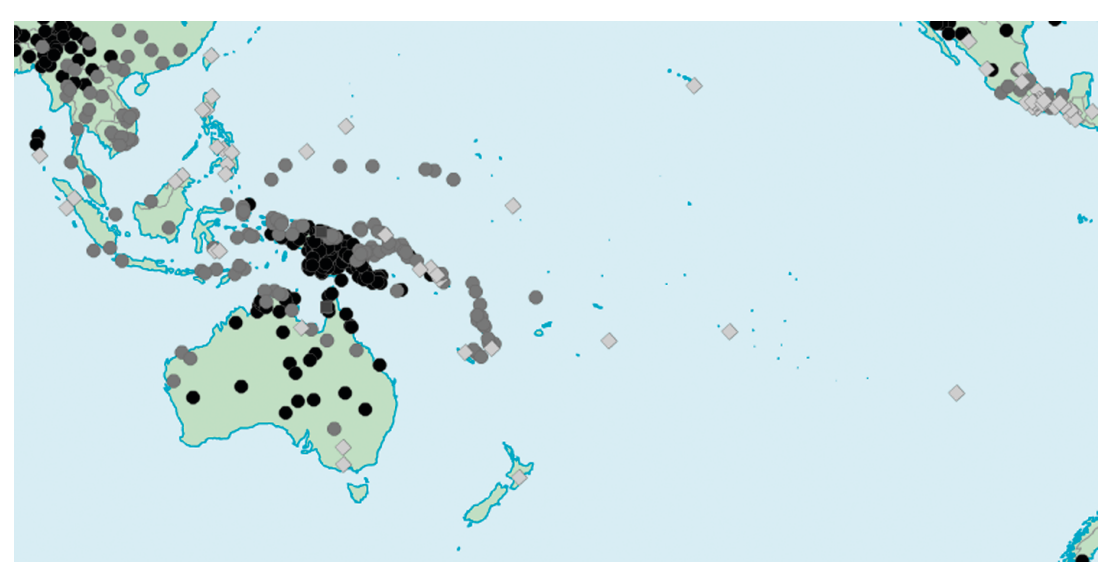

Map 2. Austronesian and non-Austronesian languages from WALS with SOV order (black)

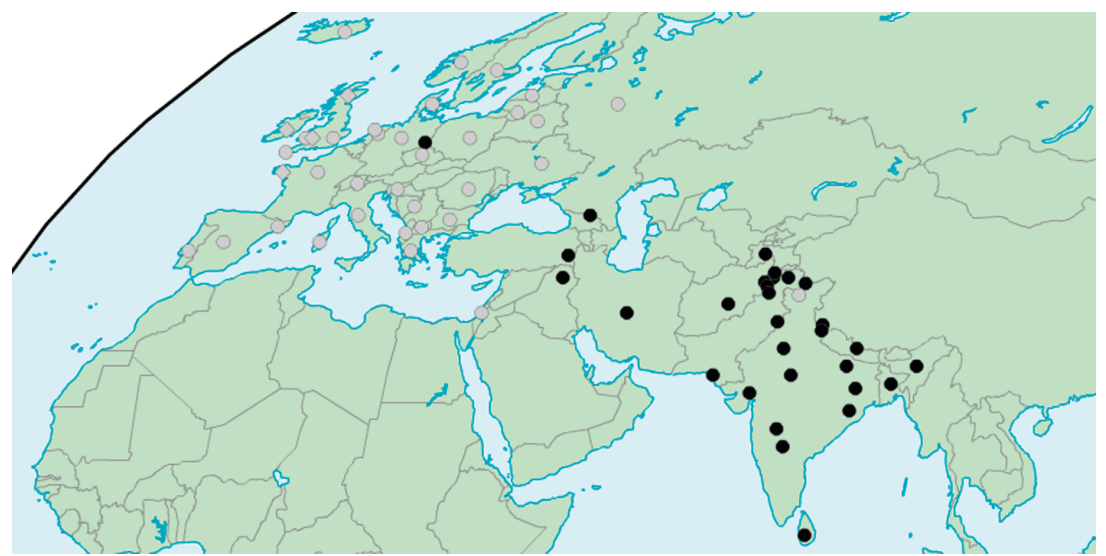

Map 3. SOV order amongst the modern Indo-European languages (black) compared to other orders (grey)

novation of SOV order amongst the Austronesian languages of mainland New Guinea is easily explained by reference to contact with (or substratal influences from) the non-Austronesian languages of the region.

Map 3 shows the occurrence of SOV order in Indo-European languages found in WALS. As with the appearance of this order in Austronesian languages, the distribution of this feature is highly skewed. In Indo-European languages in the WALS sample it is found only amongst Indo-Aryan languages of South Asia, Iranian languages, and in Sorbian. As with the Austronesian 


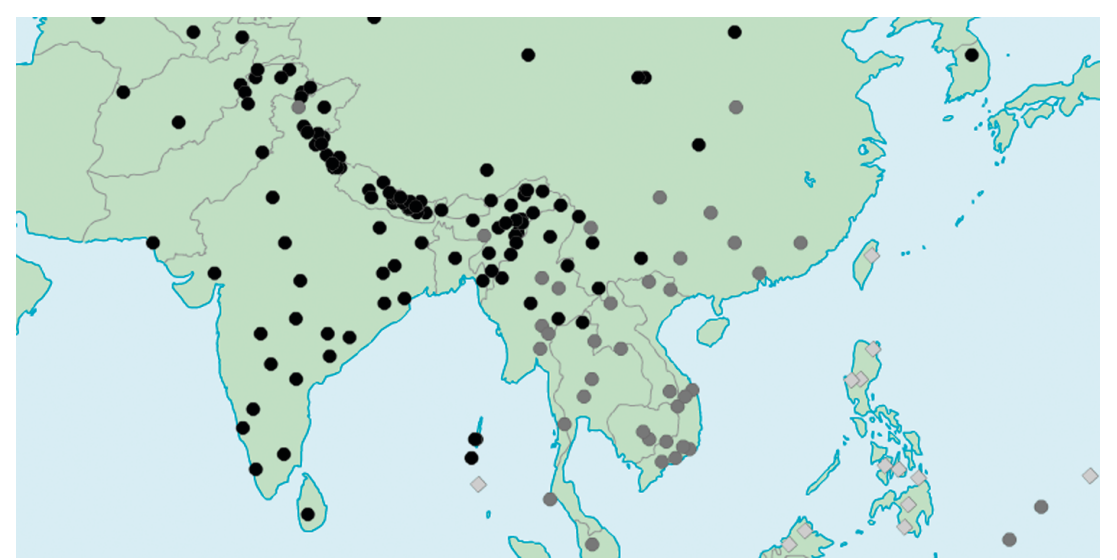

Map 4. Details of word orders in South Asia, showing all languages in Dryer 2011 $($ black $=S O V$, grey $=$ other $)$

case, I argue that this is far from a random distribution, given the areal support that exists in South Asia from languages of the non-Indo-European families to maintain an SOV order, shown in Map 4. We note that Hittite and Tocharian, not shown in Map 3, were also located in areas with a strong predominance of SOV languages, meaning that the distribution of SOV order in Indo-European has, with the exception of Sorbian (Dunn et al.'s "Lusatian", a language which - contra Dunn et al. - shows V2 order, almost certainly under the influence of German, as well as SOV order), a purely geographical explanation (though see the conclusions).

\section{A simpler model}

Stability among traits in known phylogenies can be quantified relatively simply. If we have a good idea of the reconstruction of the proto-language, and good subgrouping hypotheses, all that is required is to calculate the proportion of modern languages that attest the same value for a particular feature as in the proto-language. For instance, given SOV order in Proto-Indo-European, and a hypothesis with ten first-order subgroups, the infrequency of SOV order tells us that it is not stable. On the other hand, in the Austronesian family, reconstructed with verb-initial order, the fact that verb-initial orders are found in all major subgroups down to Proto-Oceanic means that this feature has been relatively stable in Austronesian. Wichmann \& Holman (2009) offer some more sophisticated approaches to this question, using a much greater range of data than that employed by Dunn et al. 
If a "quantitative phylogeny" is essential to the method, then the phylogeny can be quantified: how many sound changes, morphological innovations, lexical replacements occur at each node in the tree? This kind of material can be sourced from the standard historical linguistics literature (and where it is not apparent WHAT constitutes the evidence for a subgroup, we have evidence for a non-monophyletic clade). If the methods requires a binary tree, then the method is at fault unless good reasons can be shown for believing in a strictly binary linguistic history. There are no a priori reasons to assume that language families diverge in a binary fashion, and every reason to think that small, preindustrial societies existed in more of a slowly devolving dialect chain than in anything resembling a strict branching structure.

If we escape from the straitjacket that makes us believe that models of vertical (that is, direct) inheritance predominate in languages, refusing to admit that the horizontal acquisition of traits (through borrowing, or substrate effects) plays as strong a role in determining the typological and lexical make-up of a language (hence the reliance of comparative linguistics on methods other than the typological and the lexical; see, e.g., Campbell \& Poser 2008 for one recent textbook, and compare with Meillet 1908 for a seasoned account of the problems), then we are free to examine other models. Donohue \& Whiting 2011 present a statistically objective methodology for determining the distribution of linguistic traits, but visual inspection of the maps in Section 3 is just as effective in determining whether areality plays a role in the distribution of a trait or not. Given earlier debate about the diffusion of the kinds of features that Dunn et al. examine in this journal and elsewhere (e.g., Donohue \& Musgrave 2007, Holman et al. 2007, Donohue et al. 2008, Donohue et al. 2011), it is remarkable that contact has still not emerged as a possible explanation in the interpretation of computational models such as presented in Dunn et al. 2011.

Received: 31 May 2011

Australian National University

Revised: 18 July 2011

Correspondence address: Linguistics, College of Asia \& the Pacific, Australian National University, Canberra ACT 0200, Australia; e-mail: mark@donohue.cc

Acknowledgements: My thanks to the three anonymous reviewers of this article who offered critical, contentful, and valuable comments.

\section{References}

Adelaar, K. Alexander. 2005a. The Austronesian languages of South East Asia and Madagascar: A historical perspective. In K. Alexander Adelaar \& Nikolaus Himmelmann (eds.), The Austronesian languages of South East Asia and Madagascar, 1-41. London: Routledge.

Adelaar, K. Alexander. 2005b. Malayo-Sumbawan. Oceanic Linguistics 44. 357-388. 
Blust, Robert. 1981. The reconstruction of Proto-Malayo-Javanic: An appreciation. Bijdragen tot de Taal-, Land-en Volkenkunde 137. 456-469.

Blust, Robert. 2009. The Austronesian languages (Pacific Linguistics 602). Canberra: Australian National University.

Campbell, Lyle. 1997. American Indian languages: The historical linguistics of Native America. Oxford: Oxford University Press.

Campbell, Lyle \& William J. Poser. 2008. Language classification: History and method. Cambridge: Cambridge University Press.

Cavalli-Sforza, Luigi Luca \& William S.-Y. Wang. 1986. Spatial distance and lexical replacement. Language 62. 38-55.

Donohue, Mark. 2005. Word order in New Guinea: Dispelling a myth. Oceanic Linguistics 44. $527-536$.

Donohue, Mark. 2007. Word order in Austronesian: From north to south and west to east. Linguistic Typology 11. 351-393.

Donohue, Mark. 2010. The Papuanness of Papua New Guinea's eastern highlands. In Loren Billings \& Nelleke Goudswaard (eds.), Piakandatu ami Dr. Howard P. McKaughan, 87-93. Manila: Linguistic Society of the Philippines and SIL Philippines.

Donohue, Mark \& Tim Denham. 2010. Farming and language in Island Southeast Asia: Reframing Austronesian history. Current Anthropology 51(2). 223-256.

Donohue, Mark, Tim Denham \& Stephen Oppenheimer (forthcoming). Uncoupling inheritance and diffusion: A lexical-based methodology detects social distance. Diachronica.

Donohue, Mark \& Simon Musgrave. 2007. Typology and the linguistic macro-history of island Melanesia. Oceanic Linguistics 46. 348-387.

Donohue, Mark, Simon Musgrave, Bronwen Whiting \& Søren Wichmann. 2011. Typological feature analysis models linguistic geography. Language 87. 369-383.

Donohue, Mark \& Bronwen Whiting. 2011. Quantifying areality: A study of prenasalisation in Southeast Asia and New Guinea. Linguistic Typology 15. 101-121.

Donohue, Mark, Søren Wichmann \& Mihai Albu. 2008. Typology, areality and diffusion. Oceanic Linguistics 47. 223-232.

Dryer, Matthew S. 2011. Order of subject, object and verb. In Matthew S. Dryer \& Martin Haspelmath (eds.), The world atlas of language structures online, Chapter 81. München: Max Planck Digital Library. http://wals.info/chapter/81 (accessed on 30 May 2011)

Dunn, Michael, Simon J. Greenhill, Stephen C. Levinson \& Russell D. Gray. 2011. Evolved structure of languages shows lineage-specific trends in word-order universals. Nature 473. 79-82.

Foley, William A. 1986. The Papuan languages of New Guinea. Cambridge: Cambridge University Press.

Gabelentz, Georg von der. 1901. Die Sprachwissenschaft: Ihre Aufgaben, Methoden und bisherigen Ergebnisse. 2nd edn. Leipzig: Tauchnitz.

Garrett, Andrew. 2006. Convergence in the formation of Indo-European subgroups: Phylogeny and chronology. In Peter Forster \& Colin Renfrew (eds.), Phylogenetic methods and the prehistory of languages, 139-151. Cambridge: McDonald Institute for Archaeological Research.

Goddard, Ives. 1999. Native languages and language families of North America. Wall map. Lincoln, NE: University of Nebraska Press.

Goebl, Hans. 1984. Dialektometrische Studien. Anhand italoromanischer, rätoromanischer und galloromanischer Sprachmaterialien aus AIS und ALF (Beihefte zur Zeitschrift für Romanische Philologie 191-193). 3 vols. Tübingen: Niemeyer.

Grant, Anthony. 2005. The effects of intimate multidirectional linguistic contact in Chamic. In Anthony Grant \& Paul Sidwell (eds.), Chamic and beyond: Studies in mainland Austronesian languages (Pacific Linguistics 569), 37-104. Canberra: Australian National University.

Gray, Russell D., Alexei J. Drummond \& Simon J. Greenhill. 2009. Language phylogenies reveal expansion pulses and pauses in Pacific settlement. Science 323. 479-483. 
Hess, Thom. 1979. Central Coast Salish words for deer: Their wavelike distribution. International Journal of American Linguistics 45. 5-16.

Holman, Eric W., Christian Schultze, Dietrich Stauffer \& Søren Wichmann. 2007. On the relation between structural diversity and geographical distance among languages: Observations and computer simulations. Linguistic Typology 11. 395-423.

Holman, Eric W., Søren Wichmann \& Cecil Brown (no date). Inheritance and diffusion of language and culture: A comparative perspective. Available online at http://email.eva.mpg.de/ $\sim$ wichmann/wichmann_publ.html (accessed on 30 May 2011)

Kessler, Brett. 2001. The significance of word lists. Stanford, CA: CSLI Publications.

Lynch, John, Malcolm Ross \& Terry Crowley. 2002. The Oceanic languages. London: Routledge. Marck, Jeff. 2000. Topics in Polynesian language and culture history (Pacific Linguistics 504). Canberra: Australian National University.

Meillet, Antoine. 1908. Les dialectes indo-européens. Paris: Champion.

Mithun, Marianne. 1999. The languages of Native North America. Cambridge: Cambridge University Press.

Nakhleh, Luy, Don Ringe \& Tandy Warnow. 2005. Perfect phylogenetic networks: A new methodology for reconstructing the evolutionary history of natural languages. Language 81. 382420.

Pawley, Andrew K. 1972. On the internal relationships of Eastern Oceanic languages. In Roger Green \& Marion Kelly (eds.), Studies in Oceanic culture history, Vol. 3, 1-142. Honolulu: Bishop Museum.

Ringe, Don, Tandy Warnow \& Ann Taylor. 2002. Indo-European and computational cladistics. Transactions of the Philological Society 100. 59-129.

Ross, Malcolm D. 1988. Proto-Oceanic and the Austronesian languages of Western Melanesia (Pacific Linguistics C-98). Canberra: Australian National University.

Ross, Malcolm D. 1995. Some current issues in Austronesian linguistics. In Darrell T. Tryon, Malcom Ross, Adrian Clynes, Charles Grimes \& Alexander Adelaar (eds.), Comparative Austronesian dictionary: An introduction to Austronesian studies, Vol. 1(1), 45-120. Berlin: Mouton de Gruyter.

Ross, Malcolm D. 2001. Contact-induced change in Oceanic languages in North-West Melanesia. In Alexandra Aikhenvald \& R. M.W. Dixon (eds.), Areal diffusion and genetic inheritance: Problems in comparative linguistics, 134-166. Oxford: Oxford University Press.

Ross, Malcolm D. 2011. Proto-Oceanic *kw. Oceanic Linguistics 50. 25-49.

Séguy, Jean 1971. La relation entre la distance spatiale et la distance lexicale. Revue de Linguistique Romane 35. 335-357.

Thomason, Sarah Grey \& Terrence Kaufman. 1988. Language contact, creolization, and genetic linguistics. Los Angeles: University of California Press.

Wichmann, Søren \& Eric Holman. 2009. Assessing temporal stability for linguistic typological features. München: Lincom Europa. 